\title{
MMHT2014 PDFs - HERA I+II data and other updates.
}

\section{R.S. Thorne*}

Department of Physics and Astronomy, University College London, WC1E 6BT, UK

E-mail: robert.thorne@ucl.ac.uk

\section{L.A. Harland-Lang}

Department of Physics and Astronomy, University College London, WC1E 6BT, UK

E-mail: l.harland-lang@ucl.ac.uk

\section{A.D. Martin}

Institute for Particle Physics Phenomenology,

University of Durham, DH1 3LE, UK

E-mail: A.D.Martinedurham.ac.uk

We will cover a number of topics: some clarifications on MMHT PDFs with varying quark masses; a review of the fit to final HERA data, with consequences for MMHT PDFs, and a study of the fit quality; a brief introduction to topics just starting investigation, mainly the inclusion of new LHC data sets.

XXIV International Workshop on Deep-Inelastic Scattering and Related Subjects

11-15 April, 2016

DESY Hamburg, Germany

\footnotetext{
*Speaker.
} 
The MMHT2014 PDFs [1] have now superseded the previous MSTW2008 PDFs [2], due to a number of developments in theory or procedures and also a wide variety of new data sets included in the fit, many from the LHC. Other changes in data included were the replacement of the HERA run I neutral and charged current data provided separately by $\mathrm{H} 1$ and ZEUS with the combined HERA data set [3] and inclusion of HERA combined data on $\sigma^{c \bar{c}}\left(x, Q^{2}\right)$ [4]. This new set of PDFs was followed by a study of the $\alpha_{S}\left(M_{Z}^{2}\right)$ dependence [5], and most recently of the heavy quark masses dependence [6]. We briefly highlight some features of the latter.

As for MSTW2008 PDFs, we made the standard MMHT PDFs sets (i.e. exactly the same input at $Q_{0}^{2}=1 \mathrm{GeV}^{2}$ ) available for three flavour and four flavour fixed-flavour number schemes (FFNS). As default we fix the number of flavours in the running of $\alpha_{S}$, but we also provide analogous sets with variable flavour $\alpha_{S}$ for $n_{f}=4$ as there were some requests for this for MSTW2008. We have also made available sets with fits performed for $m_{c}$ and $m_{b}$ (defined in pole scheme) varying from default values of $m_{c}=1.40 \mathrm{GeV}$ and $m_{b}=4.75 \mathrm{GeV}$ in steps of $0.05 \mathrm{GeV}$ and $0.25 \mathrm{GeV}$ respectively. We might expect $m_{c}^{\text {pole }}=1.5 \pm 0.2 \mathrm{GeV}$ and $m_{b}^{\text {pole }}=4.9 \pm 0.2 \mathrm{GeV}$ from the conversion of $m_{b}$ from the $\overline{M S}$ definition and $m_{b}^{\text {pole }}-m_{c}^{\text {pole }}=3.4 \mathrm{GeV}$ with a very small uncertainty $[7,8]$ due to renormalon ambiguity cancellation. In fact $m_{b}$ is constrained to fairly close to $m_{b}=$ $4.75 \mathrm{GeV}$ from direct $\sigma^{b \bar{b}}$ data from HERA, and $m_{c}$ is also constrained from various sources. When we perform global fits with varying quark masses there is a preference for $m_{c} \sim 1.25 \mathrm{GeV}$, which is low, but not markedly inconsistent with our default. This is similar if $\alpha_{S}\left(M_{Z}^{2}\right)$ is fixed at 0.118 or left free, when a best fit value of 0.1167 is preferred. The prediction using our default $m_{b}=$ $4.75 \mathrm{GeV}$ for $\sigma^{b \bar{b}}$ data is good, as seen in Fig. 7 of [6], though a slightly lower mass is preferred. However, we note that for both $\sigma^{c \bar{c}}$ and $\sigma^{b \bar{b}}$ details on mass dependence and absolute values of $\chi^{2}$ depend on choice of multiplicative or additive definition of correlated uncertainties. For $\sigma^{c \bar{c}}$ the $\chi^{2}$ is lower and the difference between NLO and NNLO less if the additive rather than the default multiplicative definition is used. For both sets of data the additive definition pushes the preferred mass a little higher. The variation of cross sections with quark masses using $\Delta m_{c}= \pm 0.15 \mathrm{GeV}$ and $\Delta m_{b}= \pm 0.5 \mathrm{GeV}$ is shown in tables 3-5 of [6]. They are small but not insignificant - e.g. for the Higgs cross section via gluon fusion at $14 \mathrm{TeV}$ they are about $0.5 \%$ for $\Delta m_{c}$ (much less for $\Delta m_{b}$ ), about a third the PDF-only uncertainty. Changes are easily understood from the PDF behaviour with changing quark masses. We suggest adding these small uncertainties in quadrature with other sources. As an extreme example of variation with $m_{c}$, the change in $\sigma_{g g \rightarrow H}$ at $14 \mathrm{TeV}$ for $m_{c}=1.15-1.55 \mathrm{GeV}$ and free $\alpha_{S}$ is $41.01-42.28 \mathrm{pb}$ when PDF and quark mass correlations are taken into account, and part of this is actually due to the $\alpha_{S}$ variation.

We did not include separate run II H1 and ZEUS cross section data sets in the MMHT2014 fit since it was clear the run I + II combined data would soon appear. The data, and the accompanying HERAPDF2.0 analysis, are now available in [9]. It was possible that these data might produce a significant change in the PDFs and their uncertainties, and it was a high priority to investigate their impact [10]. Using $Q_{\min }^{2}=2 \mathrm{GeV}^{2}$ there are 1185 data points with 162 correlated systematics (and 7 procedural uncertainties), separated into 7 subsets, depending on whether it is $e^{+}$or $e^{-}$ beams, neutral or charged current scattering and on the proton beam energy $E_{p}$. This compared to 621 data points, separated into 5 subsets, from the HERA I combined data used previously. We first investigated the fit quality from the predictions using MMHT2014 PDFs. This is already rather good, i.e. $\chi_{N L O}^{2} / N_{p t s}=1611 / 1185=1.36$ and $\chi_{N N L O}^{2} / N_{p t s}=1503 / 1185=1.27$. In contrast 
HERAPDF2.0 PDFs, which are fit to only these data, obtain $\sim 1.20$ per point with $Q_{\min }^{2}=2 \mathrm{GeV}^{2}$ at both orders. Next we replaced the previous HERA run I data with the new combined data in the MMHT2014 fit. The fit improves to $\chi_{N L O}^{2} / N_{p t s}=1533 / 1185=1.29$, with $\Delta \chi^{2}=29$ for other data, and $\chi_{N N L O}^{2} / N_{p t s}=1457 / 1185=1.23$, with $\Delta \chi^{2}=12$ in other data, a significant, but not dramatic improvement. For direct comparison with the HERAPDF2.0 study we also fit only HERA run I + II data (we fix 4 of our PDF parameters, associated with the strange quark, in order to avoid particularly unusual PDFs). The result is $\chi_{N L O}^{2} / N_{p t s}=1416 / 1185=1.19$ and $\chi_{N N L O}^{2} / N_{p t s}=$ $1381 / 1185=1.17$. Hence, as for the global fit, the NNLO fit quality is better than NLO. Part of this is due to the charged current data $\chi^{2}$ which is about 20 units better in HERA data only fits than the global fits and about 10 units better at NNLO than at NLO. There is clear tension between these charged current data and other data in the global fit, though this less marked at NNLO.
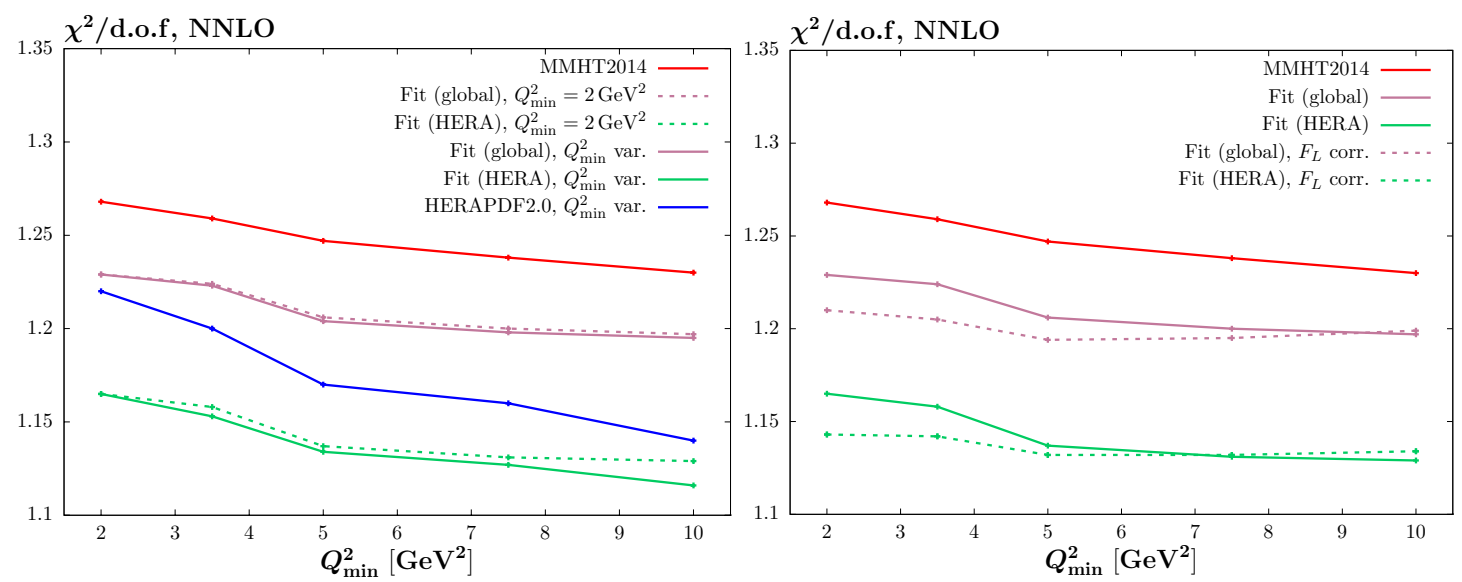

Figure 1: The variation in $\chi^{2}$ per point when increasing the $Q_{\min }^{2}$ cut, on only HERA Run I + II data, at NNLO (left) and with higher twist $F_{L}\left(x, Q^{2}\right)$ correction (right). Figures from [10].

The HERAPDF2.0 analysis saw an improvement in $\chi^{2}$ per point with a raising of the $Q_{\min }^{2}$ value. Hence, we looked at the variation of the fit quality with $Q_{\min }^{2}-$ first the quality of the comparison to data as a function of $Q_{\min }^{2}$ at NLO and at NNLO without refitting, and then after a refit. This is shown for NNLO (NLO is very similar) in the left of Fig. 1 where we have a comparison of $\chi^{2}$ for the three variations at NNLO; MMHT2014 prediction, global refit with new HERA data, and refit with only HERA run I + II combined data. We note that while there is an improvement in $\chi^{2}$ per point with increasing $Q_{\min }^{2}$ this is very largely achieved without any refitting. There is also less improvement in our analysis than for HERAPDF2.0, particularly in the global fit. In order to investigate the source of the improvement with increasing $Q_{\min }^{2}$ we looked in more detail at the fit quality for low $Q^{2}$ bins. We found that the lowest $x$ point at each $Q^{2}$ is often below theory, i.e. there is evidence of more of a turn-over in data than theory.

In Fig. 2 we show the central values of the NNLO PDFs from the fits including the new HERA combined data, comparing them to MMHT2014 PDFs (with uncertainties) and the HERAPDF2.0 PDFs (also with uncertainties). The refit PDFs are always very well within the MMHT2014 uncertainty bands. When fitted $\alpha_{S}\left(M_{Z}^{2}\right)=0.1172-3$ at NNLO, i.e. no real change from the MMHT2014 value of 0.1172 . The PDFs from the HERA run I + II data only fit are in some ways similar to those of HERAPDF2.0, e.g. the up valence quark for $x>0.2$, which shows some significant deviations from the two global fit PDF sets due to the pull of the $e^{-}$charged current data. However, 

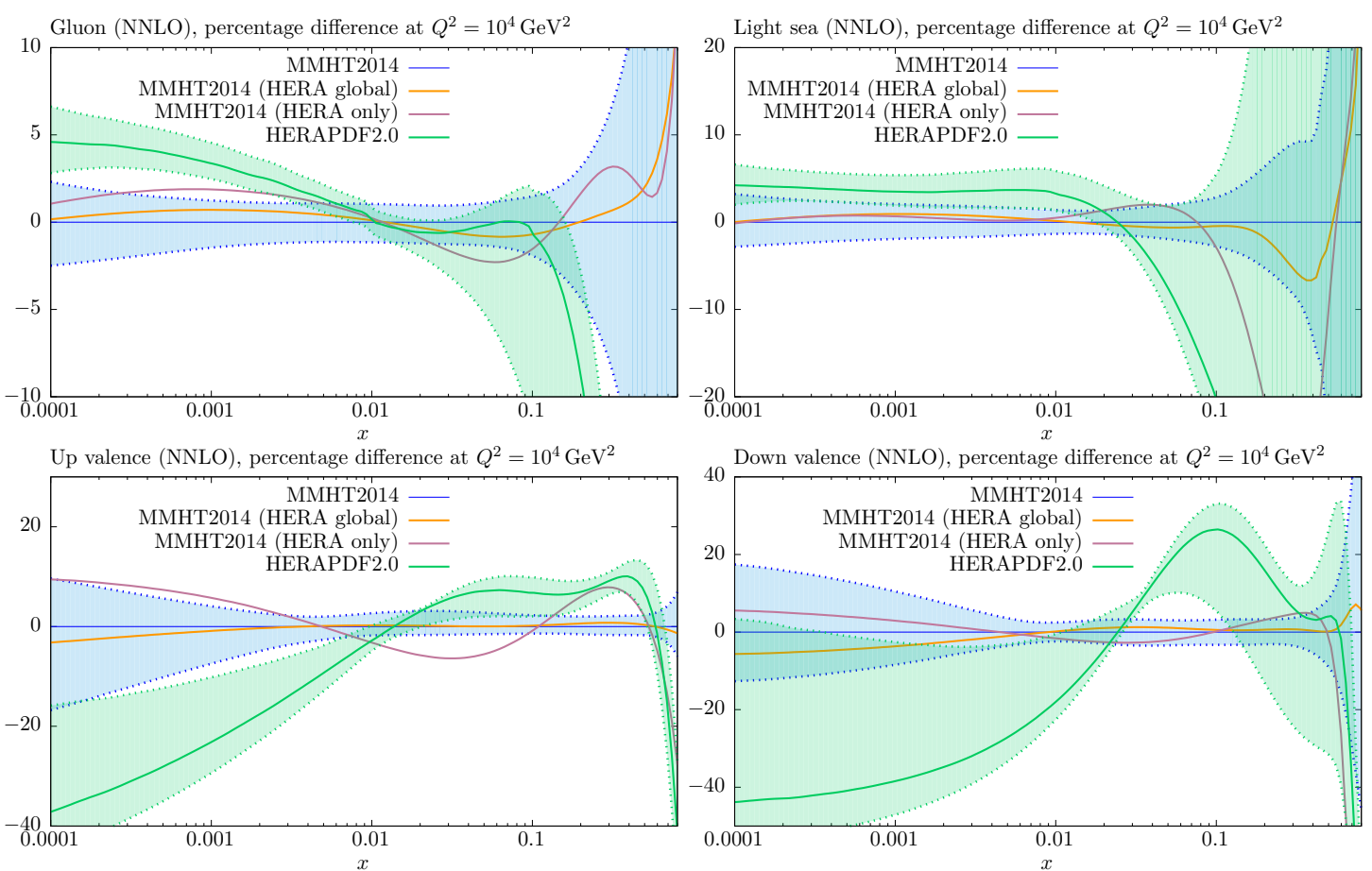

Figure 2: The comparison of NNLO MMHT PDFs containing the new HERA data to MMHT2014 PDFs and the HERAPDF2.0 PDFs. Figures from [10].

the features are far from universal. The gluon and the down valence are much more similar to MMHT2014 than HERAPDF2.0. This is likely a feature of the differing parameterisations used in the two studies. We also investigate the effect of the new data on the uncertainties of the PDFs. The most obvious improvement is to the gluon for $x<0.01$. There is also slight improvement in some places for the valence quarks. The effect is more obvious when looking at predictions, as seen in Table 1. We note that NNPDF results on central values and uncertainties are similar [11]. Hence, the HERA run I + II combined data gives us our single best constraint on PDFs, and determines the gluon at low $x$ even more accurately than before, but its inclusion does not suggest updates of existing PDFs are immediately necessary.

\begin{tabular}{|c|c|c|}
\hline & MMHT14 & MMHT14 (HERA global) \\
\hline$W^{+} \mathrm{LHC}(7 \mathrm{TeV})$ & $6.197_{-0.092}^{+0.103}\left({ }_{-15 \%}^{+1.7 \%}\right)$ & $6.221_{-0.096}^{+0.100}\left({ }_{-15 \%}^{+1.6 \%}\right)$ \\
\hline$W^{-} \mathrm{LHC}(7 \mathrm{TeV})$ & $4.306_{-0.076}^{+0.067}\left(\begin{array}{l}+1.6 \% \\
-1.8 \%\end{array}\right)$ & $4.320_{-0.070}^{+0.064}\left(\begin{array}{l}+1.5 \% \\
-1.6 \%\end{array}\right)$ \\
\hline$Z \mathrm{LHC}(7 \mathrm{TeV})$ & $0.964_{-0.013}^{+0.014}\left(\begin{array}{l}+1.5 \% \\
-1.3 \%\end{array}\right)$ & $0.966_{-0.013}^{+0.015}\left(\begin{array}{l}+1.6 \% \\
-1.3 \%\end{array}\right)$ \\
\hline$W^{+} \mathrm{LHC}(14 \mathrm{TeV})$ & $12.48_{-0.18}^{+0.22}\left(\begin{array}{l}+1.8 \% \\
-1.4 \%\end{array}\right)$ & $12.52_{-0.18}^{+0.22}\left({ }_{-1.4 \%}^{+1.8 \%}\right)$ \\
\hline$W^{-} \mathrm{LHC}(14 \mathrm{TeV})$ & $9.32_{-0.14}^{+0.15}\left(\begin{array}{c}+1.6 \% \\
-1.5 \%\end{array}\right)$ & $9.36_{-0.13}^{+0.14}\left({ }_{-1.4 \%}^{+1.5 \%}\right)$ \\
\hline$Z \mathrm{LHC}(14 \mathrm{TeV})$ & $2.065_{-0.030}^{+0.035}\left(\begin{array}{l}+1.7 \% \\
-1.5 \%\end{array}\right)$ & $2.073_{-0.026}^{+0.036}\left(\begin{array}{l}+1.7 \% \\
-1.3 \%\end{array}\right)$ \\
\hline Higgs LHC (7 TeV) & $14.56_{-0.29}^{+0.21}\left(\begin{array}{l}+1.4 \% \\
-2.0 \%\end{array}\right)$ & $14.52_{-0.24}^{+0.19}\left(\begin{array}{l}+1.3 \% \\
-1.7 \%\end{array}\right)$ \\
\hline Higgs LHC (14 TeV) & $47.69_{-0.88}^{+0.63}\left(\begin{array}{l}+1.3 \% \\
-1.8 \%\end{array}\right)$ & $47.75_{-0.72}^{+0.59}\left(\begin{array}{l}+1.2 \% \\
-1.5 \%\end{array}\right)$ \\
\hline$t \bar{t} \mathrm{LHC}(7 \mathrm{TeV})$ & $175.9_{-5.5}^{+3.9}\left(\begin{array}{c}+3.1 \% \\
+2.2 \%\end{array}\right)$ & $174.8_{-5.3}^{+3.3}\left({ }_{-3.0 \%}^{+1.9 \%}\right)$ \\
\hline$t \bar{t} \mathrm{LHC}(14 \mathrm{TeV})$ & $970_{-20}^{+16}\left(\begin{array}{l}+1.6 \% \\
-2.1 \%\end{array}\right)$ & $964_{-19}^{+13}\left(\begin{array}{l}+1.3 \% \\
-2.0 \%\end{array}\right)$ \\
\hline
\end{tabular}

Table 1: The values of various cross sections (in nb) obtained with the NNLO MMHT 2014 sets, with and without the final HERA combination data set included. PDF uncertainties only are shown. 
In order to try to minimise the tendency to overshoot high $y$ points at low $x$ and $Q^{2}$, we tried a modification $F_{L} \rightarrow\left(1+A / Q^{2}\right) F_{L}$ for $x<0.01$. This leads to $\Delta \chi^{2}=-24$ for $Q_{\min }^{2}=2 \mathrm{GeV}^{2}$ and $A \approx 4 \mathrm{GeV}^{2}$. A very similar result is found in a fit to only HERA data. Adding $\left(B / Q^{4}\right) F_{L}$ leads to little further improvement and additional corrections $F_{2} \rightarrow\left(1+A_{i} / Q^{2}\right) F_{2}$ for $x<0.01$ lead to $A_{i} \sim 0.1$, but with low significance, and to further $\Delta \chi^{2}=-10$, almost all for non HERA data. The best fit is a big change in $F_{L}\left(x, Q^{2}\right)$ at small $x$ and $Q^{2}$, but this is a region where $F_{L}\left(x, Q^{2}\right)$ varies quickly from one order to the next and is sensitive to many potential corrections. The results of the fits with the $F_{L}\left(x, Q^{2}\right)$ modification are seen in the right of Fig. 1, and just about all evidence of a fall of $\chi^{2}$ per point with $Q_{\min }^{2}$ is eliminated. Similar results have been found recently in [12]. The PDFs with higher twist $F_{L}\left(x, Q^{2}\right)$ corrections are extremely similar to those without. We also tried an alternative correction of the form $F_{L}\left(x, Q^{2}\right)\left(1+\frac{\alpha_{S}\left(Q^{2}\right)}{4 \pi} \frac{b_{1}}{x^{b_{2}}}\right)$, leading to $\rightarrow \Delta \chi^{2}=-28$ with $b_{1}=0.014$ and $b_{2}=0.82$. However, as at fixed high $y$, where $F_{L}\left(x, Q^{2}\right)$ is important, we have $x \propto Q^{2}$, the power of $b_{2} \sim 1$ in combination with the slow falling of $\alpha_{S}\left(Q^{2}\right)$ leads to the correction being effectively $\sim 1 / Q^{2} \cdot \ln (1 / x)$ terms were less successful than the power term. If we try a correction of the form $F_{L}\left(x, Q^{2}\right)\left(1+c_{1} x^{c_{2}}\right)$ then $\Delta \chi^{2}=-13$ with $c_{1}=-1.97$ and $c_{2}=0.42$, i.e. a negative correction to $F_{L}\left(x, Q^{2}\right)$ concentrated at high $x$, and hence high $Q^{2}$. Detailed examination of data against theory shows that the predictions at high $Q^{2}$ and high $y$ show a tendency to undershoot the data - the opposite trend to the low $Q^{2}$ case. An addition of both type of term allows an overall $\rightarrow \Delta \chi^{2}=-42$, even better than the sum of the two independent effects.
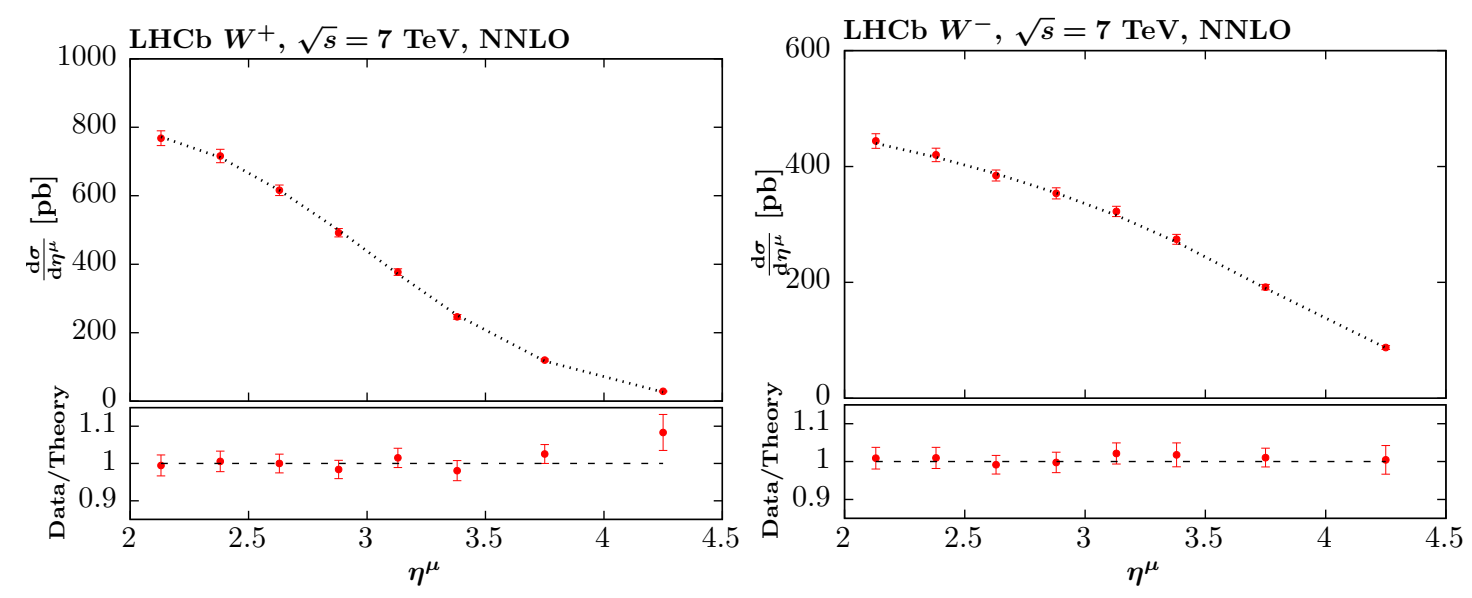

Figure 3: The comparison of predictions using the NNLO MMHT 2014 PDFs to the data on $W$ production at high rapidity in [13].

Future updates of PDFs will include new more precise data, mainly from the LHC, and work is underway. However, we note that no data so far implies a major change in the MMHT2014 PDFs. We showed a number of good predictions for data not included in the fit in [1], e.g. $W+c$ data [15] which constrains the strange quark. Additional comparisons have been made to data, for example, that in $[13,14,16]$. In most cases very good agreement is seen, e.g the comparison to $W$ data in [13] is seen in Fig. 3, particularly when the PDF uncertainty is taken into account. However, some LHC data with increased precision, e.g. that in $[14,16]$ will clearly lead to some moderate changes in central values and to a reduction in uncertainties, and a study of this is currently ongoing. Work is also progressing on an update of PDFs with QED corrections, intending to replace the photon PDF obtained in [17], where this time the input photon will have contributions from both coherent 
and incoherent photon radiation, as in [18].

\section{Acknowledgements}

We thank the Science and Technology Facilities Council (STFC) for support via grant award ST/L000377/1.

\section{References}

[1] L. A. Harland-Lang, A. D. Martin, P. Motylinski and R. S. Thorne, Eur. Phys. J. C 75 (2015) no.5, 204 doi:10.1140/epjc/s10052-015-3397-6 [arXiv:1412.3989 [hep-ph]].

[2] A. D. Martin, W. J. Stirling, R. S. Thorne and G. Watt, Eur. Phys. J. C 63 (2009) 189 doi:10.1140/epjc/s10052-009-1072-5 [arXiv:0901.0002 [hep-ph]].

[3] F. D. Aaron et al. [H1 and ZEUS Collaborations], JHEP 1001 (2010) 109 doi:10.1007/JHEP01(2010)109 [arXiv:0911.0884 [hep-ex]].

[4] H. Abramowicz et al. [H1 and ZEUS Collaborations], Eur. Phys. J. C 73 (2013) no.2, 2311 doi:10.1140/epjc/s10052-013-2311-3 [arXiv:1211.1182 [hep-ex]].

[5] L. A. Harland-Lang, A. D. Martin, P. Motylinski and R. S. Thorne, Eur. Phys. J. C 75 (2015) no.9, 435 doi:10.1140/epjc/s10052-015-3630-3 [arXiv:1506.05682 [hep-ph]].

[6] L. A. Harland-Lang, A. D. Martin, P. Motylinski and R. S. Thorne, Eur. Phys. J. C 76 (2016) no.1, 10 doi:10.1140/epjc/s10052-015-3843-5 [arXiv:1510.02332 [hep-ph]].

[7] C. W. Bauer, Z. Ligeti, M. Luke, A. V. Manohar and M. Trott, Phys. Rev. D 70 (2004) 094017 doi:10.1103/PhysRevD.70.094017 [hep-ph/0408002].

[8] A. H. Hoang and A. V. Manohar, Phys. Lett. B 633 (2006) 526 doi:10.1016/j.physletb.2005.12.020 [hep-ph/0509195].

[9] H. Abramowicz et al. [H1 and ZEUS Collaborations], Eur. Phys. J. C 75 (2015) no.12, 580 doi:10.1140/epjc/s10052-015-3710-4 [arXiv:1506.06042 [hep-ex]].

[10] L. A. Harland-Lang, A. D. Martin, P. Motylinski and R. S. Thorne, Eur. Phys. J. C 76 (2016) no.4, 186 doi:10.1140/epjc/s10052-016-4020-1 [arXiv:1601.03413 [hep-ph]].

[11] J. Rojo [NNPDF Collaboration], PoS EPS -HEP2015 (2015) 506 [arXiv:1508.07731 [hep-ph]].

[12] I. Abt, A. M. Cooper-Sarkar, B. Foster, V. Myronenko, K. Wichmann and M. Wing, Phys. Rev. D 94 (2016) no.3, 034032 doi:10.1103/PhysRevD.94.034032 [arXiv:1604.02299 [hep-ph]].

[13] R. Aaij et al. [LHCb Collaboration], JHEP 1508 (2015) 039 doi:10.1007/JHEP08(2015)039 [arXiv:1505.07024 [hep-ex]].

[14] R. Aaij et al. [LHCb Collaboration], JHEP 1601 (2016) 155 doi:10.1007/JHEP01(2016)155 [arXiv:1511.08039 [hep-ex]].

[15] S. Chatrchyan et al. [CMS Collaboration], JHEP 1402 (2014) 013 doi:10.1007/JHEP02(2014)013 [arXiv:1310.1138 [hep-ex]].

[16] V. Khachatryan et al. [CMS Collaboration], Eur. Phys. J. C doi:10.1140/epjc/s10052-016-4293-4 [arXiv:1603.01803 [hep-ex]].

[17] A. D. Martin, R. G. Roberts, W. J. Stirling and R. S. Thorne, Eur. Phys. J. C 39 (2005) 155 doi:10.1140/epjc/s2004-02088-7 [hep-ph/0411040].

[18] A. D. Martin and M. G. Ryskin, Eur. Phys. J. C 74 (2014) 3040 doi:10.1140/epjc/s10052-014-3040-y [arXiv:1406.2118 [hep-ph]]. 